\title{
EXPERIÊNCIA E PARAMETRIZAÇÃO NO PROCESSO DE PROJETO DE APLICAÇÕES DIGITAIS INTERATIVAS
}

\author{
Experience and parameterization in the process of digital \\ interactive applications design \\ Cristiane Ellwanger', Vinicius Nunes Rocha e Souza', \\ Alexandre dos Santos Roque², Underléa Miotto Bruscato', Régio Piere da Silva'
}

RESUMO Experiências de projetistas se retratam nos protótipos por eles desenvolvidos, os quais são de suma importância para a formação de conceitos, principalmente na fase de projeto e de concepção de sistemas computacionais. Diante disso, o presente artigo tem como objetivo demonstrar como a prototipação pode se beneficiar da parametrização na concepção destes sistemas. Para isso, o processo projetual, exposto neste artigo, é estabelecido metodologicamente pelo entendimento de conceitos que permeiam os contextos experienciais e interacionais (imersão); na estruturação de ideias (ideação) que delineiam uma solução passível de implementação (prototipação) e na sua posterior validação. Como resultado, é proposta uma solução computacional constituída por um sistema embarcado e validada por testes funcionais que retratam que a modelagem paramétrica favorece o estabelecimento de relações e correlações entre os componentes utilizados na prototipação, e demonstram que a parametrização auxilia tanto na criação quanto no redesenho de sistemas computacionais interativos.

\section{PALAVRAS-CHAVE: Experiência, Parametrização, Processo de projeto.}

ABSTRACT Designers' experiences are reflected on prototypes developed by them, which are of paramount importance to the formation of concepts, especially in the design and design of computer systems phases. Therefore, this article demonstrates how prototyping can benefit from the parameter in the design of these systems. To make it happen, the design process, exposed in this article, is established methodologically by the understanding of concepts that spread through the experiential and interactive contexts (immersion), in the structuring of ideas (ideation) that delineate one solution liable to implementation (prototype) and its subsequent validation. As a result, a computational solution that consists of an embedded system is proposed, validated by functional tests that show that parametric modeling hold up the establishment of relations and correlations between the components used in prototyping, and demonstrate that the parameter assists both the creation and the redesign of interactive computer systems.

KEYWORDS: Experience, Parameterization, Design Process.

\section{How to cite this article:}

ELLWANGER, C.; SOUZA, V. N. R. S.; ROQUE, A. S.; BRUSCATO, U. M., SILVA, R. P. Gestão e Tecnologia de Projetos, São Carlos, v. 11, n. 1, p. 7-20, jan./jun. 2016.

http://dx.doi.org/10.11606/gtp.v11i1.96827

Fonte de financiamento: Coordenação de Aperfeiçoamento de Pessoal de Nivel Superior (Capes). Conflito de interesse: Declaram não haver Submetido em: 26 mar. 2015 Aceito em: 25 ago. 2015 


\section{INTRODUÇÃO}

O avanço significativo de tecnologias de informação e de comunicação remete às diferentes tecnologias com as quais as pessoas interagem cotidianamente. As transformações tecnológicas e suas consequências sociais, éticas, culturais, educacionais, ambientais, dentre outras se processam em um ritmo acelerado e dinâmico, propiciando novas formas de comunicação, interação e experiência. Além disso, evidenciam a necessidade de se buscar novos modelos, novos métodos e novas abordagens para o design em contextos interacionais (PORTUGAL, 2013).

Nesse contexto, as teorias direcionadas ao Design Experiencial (DE) salientam a importância de se projetar para a experiência e à complexidade atrelada às variáveis envolvidas nesse processo. Considerações de diversos autores ressaltam a subjetividade de contextos experienciais e demonstram seus esforços na coleta e análise dos diferentes tipos de dados provenientes da interação que se tem com as mais diferentes tecnologias e com as soluções propostas que se debruçam sobre estas (MCLELLAN, 2000; SURI, 2003; FORLIZZI et al., 2003; HAYASHI, 2013). O apontamento de técnicas para o melhor direcionamento de projetistas ou que sirvam de subsídio para que se possam aplicá-las e contemplá-las no processo de desenvolvimento de sistemas computacionais interativos, ampara-se na literatura e justifica-se devido à complexidade atrelada a contextos experienciais (JÄÄSCO et al., 2003; BUCCINI, 2008; HOWARD, 2011; GARRET, 2011; HASSENZAHL, 2013).

Salienta-se a importância das pesquisas que contemplam o DE e a sua ênfase e foco nas pessoas que se utilizam de tecnologias (usuários). Entretanto, o presente artigo tem por intuito direcionar a atenção não aos aportes teóricos que fundamentam essa perspectiva e que estão em evidência na literatura, mas aos delineamentos e práticas de design que se consolidam para alcançá-la. Isso se faz necessário, tendo em vista que um dos grandes desafios da abordagem do DE é contemplar, na prática do design, os aportes teóricos que subsidiam as características inerentes ao design (FORLIZZI, 2003).

Por este motivo, o viés experiencial (o DE), sob a práxis de projetistas, pode se revelar bastante útil quando integrado à modelagem paramétrica (Design Paramétrico - DP). Isso porque o DE ampara-se em uma abordagem mais ampla que o design tradicional ao se direcionar àa criação de experiências ao invés de se direcionar simplesmente a produtos e serviços; ao considerar a visualização de todo o ciclo de vida de um produto; ao dar ênfase no estabelecimento de uma relação com indivíduos e não com uma massa de consumidores e em sua constante preocupação em propiciar a criação de um ambiente que conecte o usuário emocionalmente, agregando valor às suas experiências de compra e uso (AIGA, 2001).

Aliado ao DE, o DP pode oferecer contribuições para que experiências sejam significativas, visto que a adoção de parâmetros em um projeto exige a definição de relações e interconexões entre elementos distintos e variados a serem utilizados e determinados pelos projetistas, contribuindo para a integração e associação entre as partes que compõe o projeto. Somados a esses aspectos, favorecem os insights criativos ao estabelecer relações e definições estruturais de um produto como parte integrante do processo de design e por permitir o estabelecimento de relações e procedimentos que aprimoram o projeto como um todo (WOODBURY, 2010).

Assim, o presente artigo tem por intuito salientar a importância da experiência de projetistas no desenvolvimento de sistemas computacionais interativos e de sua práxis no processo de projeto para a experiência do usuário, apresentando inferências que remetem à convergência e à interoperabilidade tecnológica e as contribuições da modelagem paramétrica para o processo projetual. Além disso, retrata representações de conhecimento dos projetistas, originárias deste processo e demonstra como aportes teóricos aqui formalizados se retratam em sua prática. 


\section{PROCESSO DE PROJETO PARA A EXPERIÊNCIA}

O crescimento exponencial de desenvolvimento e o uso de diversas tecnologias de informação e de comunicação direcionadas a diferentes propósitos evidenciam características cada vez mais necessárias como mobilidade, capacidade de processamento e disponibilidade de informações sem restrições de tempo e de espaço. Entretanto, pensar no desenvolvimento de tecnologias pode remeter a uma determinada situação, enquanto pensar na interação que esse processo de desenvolvimento vai gerar leva a outra, pois a todo momento se interage com diferentes tipos de tecnologias.

Assim, a experiência advém da interação, ou seja, da manipulação de recursos (sejam eles computacionais ou não), e do uso que se faz destas tecnologias. Logo, a interação preconiza no comportamento das pessoas em relação a outras pessoas e aos sistemas por elas utilizados. Tem como pressuposto a ação recíproca em que indivíduos e objetos influenciamse mutuamente. Diferencia-se de interatividade, por esta referenciar a capacidade ou o potencial que um sistema apresenta de propiciar a interação, estabelecendo-se como um pré-requisito para a mesma (FIALHO, 2001).

Sob esta perspectiva o projeto de produtos interativos usáveis requer a observância de seu contexto de uso (quem e onde os mesmos serão utilizados), além da compreensão do tipo de atividade que as pessoas realizam ao interagirem com determinado produto ou serviço (ROGERS; SHARP; PREECE, 2005; 2013). Diante disso, Garrett (2011) salienta que a experiência do usuário é definida por meio do comportamento do produto (objeto) e da forma com que o mesmo é utilizado, ou seja, advém do contato (interação) do usuário com o produto (objeto), indo além das funcionalidades e recursos a ele inerentes.

Já, na concepção de Cybis e Pereira (2010) a experiência do usuário pode ser vista como o conjunto de todos os processos, sejam eles físicos, cognitivos ou emocionais, provenientes da interação do usuário com um determinado produto/serviço, em momentos que vão desde a expectativa da interação, à interação propriamente dita e à reflexão após a interação, considerando um determinado contexto de uso, seja ele físico, social ou tecnológico. Neste contexto interacional e experiencial, em que ferramentas computacionais dão suporte ao processo de projeto de soluções, experiências de projetistas se refletem sob as soluções por eles propostas - o que, na maioria das vezes, exige uma ampla gama de conhecimentos e habilidades.

Para Benyon (2013), sistemas computacionais interativos voltam-se à exibição, ao armazenamento ou à transformação de informações passíveis de serem percebidas pelas pessoas. Fundamentam-se sob os aportes teóricos relacionados à área de IHC (Interação Humano-Computador) e requerem de projetistas conhecimentos e habilidades que envolvem o estudo e o entendimento das aspirações humanas, dos contextos nos quais uma determinada tecnologia pode se revelar útil, das possibilidades que a tecnologia escolhida pode oferecer, bem como, dos demais aspectos que envolvem o projeto de soluções tecnológicas que combinem com as pessoas, com as atividades realizadas por elas e com os contextos em que estas atividades ocorrem.

Assim, o DE delineia a experiência de projetistas na concepção de sistemas computacionais interativos. Por outro lado, o DP possibilita o aprimoramento dessa experiência, a partir do ajuste de parâmetros e das inferências que podem ser realizadas sob estes parâmetros.

\section{DESIGN PARAMÉTRICO - CONTRIBUIÇÕES}

O DP pode oferecer contribuições para que experiências sejam significativas, pois a adoção de parâmetros em um projeto exige a definição 
de relações e interconexões entre vários elementos distintos e variados. Na concepção de Woodbury (2010), a utilização do DP no processo projetual favorece a verificação de como estas partes podem ser associadas e integradas e favorecem os insights criativos.

Além disso, projetos podem ser mais bem delineados, estruturados e reestruturados quando se utilizam de parâmetros, tendo em vista que, neste caso, são considerados o projeto e suas especificidades e não a sua forma. A parametrização permite contemplar estas especificidades, à medida que parâmetros se estabelecem como elementos variáveis e quantificáveis que permitem a configuração de um objeto ou de um produto, considerando as relações existentes entre os elementos que o compõem, ou ainda, as relações existentes entre as relações estabelecidas (KOLAREVIC, 2009).

Estas características viabilizam a aplicação de técnicas digitais, as quais se estabelecem por meio de algoritmos implícitos que não se expressam numa forma específica. Os parâmetros, integrados a estes algoritmos, ampliam o campo de possibilidades formais e arquiteturais de um produto, propiciando a manipulação de relações e a constituição de geometrias associativas que não são soluções fixas. Assim, todas as partes que compõem um determinado produto correspondem a um todo integrado e se modificam de maneira sistemática e coordenada, estabelecendo conexões, onde poucos elementos que compõem um projeto podem ser modificados, sem perda de suas funcionalidades.

Em termos de programação, isso favorece a diversidade e amplia as possibilidades de se criar e recriar produtos, dado que no DP são os parâmetros de um projeto específico que são declarados e não a sua forma e no qual técnicas digitais configuram-se através de algoritmos implícitos. Assim, parâmetros podem ampliar o campo de possibilidades formais da arquitetura através da manipulação de relações, favorecendo a concepção de geometrias associativas que não são soluções fixas (WOODBURY, 2010) (KOLAREVIC, 2005, 2009).

Cada parâmetro contém um potencial real para se tornar um dado que consequentemente modifica outros parâmetros. Entretanto, são vistos como entidades únicas, irredutíveis entre si e constituem-se como a base fundamental para a constituição do processo de fabricação digital (PARISI, 2013).

\section{A IMPORTÂNCIA DA PROTOTIPAÇÃO PARA APLICAÇÕES INTERATIVAS}

Técnicas de modelagem e prototipação digital auxiliam na redução de tempo de lançamento de um determinado produto ao permitir simulações e correções rápidas no projeto (ESPINOZA; SCHAFFER, 2004) e são fatores determinantes para o sucesso do produto, pois custos com correções no desenvolvimento do projeto aumentam significativamente na medida em que as etapas do projeto avançam (BACK et al., 2008; HALLGRIMSON, 2012). Diante disso, protótipos podem ser utilizados para resolver problemas de projeto e melhorar a compreensão que se tem do produto em desenvolvimento. Dentre outros usos, eles podem auxiliar a geração de ideias, testes com o usuário, comunicação e verificação de requisitos de projeto.

$\mathrm{Na}$ geração de ideias, protótipos devem ser produzidos de forma rápida e sem preocupações com detalhes, pois o objetivo é verificar experimentalmente a viabilidade de uma proposta de solução por meio da exploração de diferentes aspectos do produto físico, como materiais, tecnologias, forma e função. Para isso, protótipos podem ser desenvolvidos em paralelo, considerando diferentes aspectos, como aparência e função. Protótipos em tamanho real também determinam o fluxo de tarefas e os 
requisitos de projeto. Assim, podem ser testados requisitos ergonômicos, tais como ajustes, medidas e questões cognitivas. Entretanto, é necessário explicitar quais testes e tipos de tarefas serão realizados pelo usuário para que um protótipo seja construído de forma adequada.

Um dos mais significativos usos do protótipo físico está na realização de testes que envolvam a participação de usuários, no entanto, eles também servem para que projetistas possam verificar se uma determinada aplicação funciona conforme o esperado, possibilitando que interferências possam ser realizadas sob ela, antes que testes com usuários sejam realizados. Ademais, protótipos físicos e digitais podem auxiliar na comunicação eficaz entre a equipe de desenvolvimento e os stakeholders (interessados e envolvidos no processo de projeto), pois esboços digitais favorecem a criação de modelos e oferecem a possibilidade de se gerar muitas variações do projeto a partir do ajuste de parâmetros geométricos. Apresentam a possibilidade de incorporar propriedades de materiais de fabricação e restrições lógicas de montagem em parâmetros, sendo possível até mesmo responder ao ambiente e ser associativo em sentido mais amplo (HENSEL; MENGES, 2008) (HALLGRIMSON, 2012).

Hensel e Menges (2008) salientam ainda que o DP permite o reconhecimento de padrões de comportamento geométrico e capacidades performativas relacionados, e tendências do sistema. No contínuo feedback com o ambiente externo, essas tendências comportamentais podem informar o desenvolvimento ontogenético de um sistema específico, através de diferenciações paramétricas e suas sub-localizações. Segundo Aish e Woodbury (2005), a indústria tem direcionado a atenção para sistemas que possibilitam a manipulação de artefatos de forma paramétrica. Isso ocorre pelo fato dessa forma de representação admitir uma rápida mudança de dimensões de concepção e de estrutura. Entretanto, a parametrização aumenta a complexidade tanto da tarefa a ser desenvolvida quanto do design de interface utilizado pelos projetistas, visto que eles modelam não apenas o artefato que está sendo projetado, mas uma estrutura conceitual orientada a variações.

Diante disso, o uso da parametrização tem impactos positivos e negativos. Na concepção de Aish e Woodbury (2005), positivos no sentido dela maximizar a busca por modelos mais adaptados ao contexto e isso pode facilitar a descoberta de novas formas e tipos de formas, reduzir o tempo e o esforço necessários para as mudanças e reutilização de um determinado artefato, além de facilitar a produção de melhores entendimentos sobre a estrutura conceitual do artefato a ser projetado. Sob o aspecto negativo, ela pode exigir esforços adicionais, considerando-se a complexidade de decisões de design que ela agrega em si.

$\mathrm{O}$ fato de variáveis independentes serem diretamente manipuladas a partir de uma interface retrata uma característica emergente de que sistemas que contemplam o DP podem, de certa forma, ser sua própria interface. Contudo, projetistas direcionam grandes esforços na modelagem de estruturas gráficas que remetem a outras estruturas gráficas. Tais padrões de consumo tem sido o impulso para o desenvolvimento de aplicações mais genéricas que, com efeito, se refletem nos elementos de componentes generativos de interfaces de usuário (AISH; WOODBURY, 2005).

Todavia, faz-se necessário compreender como as diferentes tecnologias, utilizadas no processo de fabricação digital, conectam-se entre si e como podem ser interoperáveis. A parametrização em um processo projetual não deveria limitar-se aos aspectos relacionados à gênese da forma, mas sim na importância de se definir parâmetros que definem as relações e a vontade de projetistas em considerar a observação, a representação e a performance como referências estabelecidas de modo a se tornarem partes integrantes de um processo de projeto mais amplo e colaborativo (SCHEEREN; SPERLING, 2013). 


\section{CONVERGÊNCIA E INTEROPERABILIDADE EM PROCESSOS PROJETUAIS}

Embora os sistemas computacionais estejam limitados ao conjunto de funções pré-definidas pelas "escolhas a partir das quais o próprio sistema que as enuncia foi configurado" (FRAGOSO, 2005), eles devem ser provenientes de um processo que extrapole os meandros que os deu origem, considerando uma abordagem "pragmática que não seja somente centrada em aspectos técnicos, nem no social, mas capaz de respeitar a dinâmica não hierárquica e não linear de suas relações” (LATOUR, 2001).

O objeto em si, bem como o debatido binômio forma-função já não serve mais. Na concepção de Latour, complementada por Manzini (2004), "passamos dos objetos aos projetos, da aplicação à experimentação, de um mundo sólido a um mundo fluido, pensado e construído sob a estabilidade dos objetos, cuja verdadeira essência é a variabilidade dos projetos e a dinâmica das interações”. No entendimento do autor isso desacomoda e desestabiliza a zona de conforto de designers, firmada sobre um mundo sólido do passado, no qual "containers disciplinares" seguros retratavam a posição de qualquer um, pela definição de sua própria identidade profissional.

Atualmente essas definições profissionais e disciplinares se dissolvem e qualquer um deve, continuamente, redefinir a si mesmo suas capacidades e competências (MANZINI, 2004). A variabilidade de projetos e a dinâmica das interações, exposta por Latour, referendada e complementada por Manzini (2004), são resultantes de um processo projetual que considera e aprecia a convergência e a interoperabilidade tecnológica em e entre interfaces que compõem os sistemas computacionais a serem desenvolvidos e nas quais as características físicas dos dispositivos, a forma de interação e elementos transientes, definidos em interfaces, corroboram para a constituição do sistema como um todo, o que requer uma "abordagem de modelagem que contemple as restrições de projeto em detrimento destas especificidades" (PRESSMAN; LOWE, 2009).

Ademais, faz-se necessário compreender as características e particularidades inerentes aos próprios dispositivos, seus diferentes formatos e formas de conexão. Conexões essas que estabelecem a forma com que dispositivos conectam-se com tecnologias públicas (checkouts), com diferentes tipos de dispositivos de exibição (projetores, televisores), com recursos físicos como RFID (Radio Frequency Identification), microcontroladores, NFC (Near Field Communication), bem como dispositivos que requerem padrões específicos de comunicação (wifi, bluetooth) (BENYON, 2011).

Diante desses aspectos e considerações o autor salienta que a definição e delineamento de requisitos para a concepção, o desenvolvimento, a estruturação e a reestruturação de soluções se apresentam como um grande desafio aos projetistas de sistemas computacionais interativos, pois requer além do entendimento de como pesquisar pessoas, a compreensão de como elas interagem com diferentes tipos de dispositivos, agregando-se ainda as aplicações projetadas sobre estes dispositivos. Cenários de produção no campo do design se estabelecem de forma múltipla, fluida e dinâmica, conduzem projetistas a aprender a lidar com o excesso de informação, valendo-se de novas ferramentas, instrumentos e metodologias que lhe permitam compreender e gerenciar a complexidade, característica dos contextos culturais contemporâneos (PRESSMAN; LOWE, 2009).

Assim, a partir da prototipação, projetistas se voltam à compreensão e ao entendimento de como características como interoperabilidade e convergência se apresentam nas ferramentas computacionais que lhes dão suporte ao processo projetual, considerando-se que a interoperabilidade consiste na capacidade que um sistema ou uma aplicação apresenta para interagir com outros sistemas, a um custo baixo e sob uma abordagem 
flexível (MERTINS et al., 2014). Em grande parte tais características se apresentam sob a forma de sistemas computacionais embarcados, os quais se inserem em diferentes contextos. São responsáveis por tarefas importantes, desde o controle de equipamentos domésticos ou de entretenimento até sofisticados sistemas de controle aéreo. Referenciados na literatura como sistemas embutidos ou sistemas dedicados, direcionam-se ao controle de um determinado ambiente ou dispositivo físico.

De acordo com Broekman (2003) um sistema embarcado é definido como um sistema computacional especializado que faz parte de uma máquina ou de um sistema maior, que podem ser encontrados em uma ampla variedade de equipamentos. Muitas vezes, despercebidos integram-se a artefatos eletrônicos, como celulares, relógios, televisores, equipamentos médicos, automóveis, videogames e uma série de outros produtos que moldam praticamente todas as atividades humanas, e com os baixos custos tecnológicos atuais, tendem a aumentar sua presença no cotidiano das pessoas (LI; XIONG, 2005).

O projeto de sistemas embarcados é complexo e normalmente requer processos similares aos usados no desenvolvimento de software, e da mesma forma, testes realizados sob estes sistemas devem ser exaustivos, devido ao impacto crítico que uma falha pode gerar no ambiente externo, segundo Yang (2012). De forma simplificada, a Figura 1, ilustra o diagrama de blocos de um sistema embarcado hipotético.

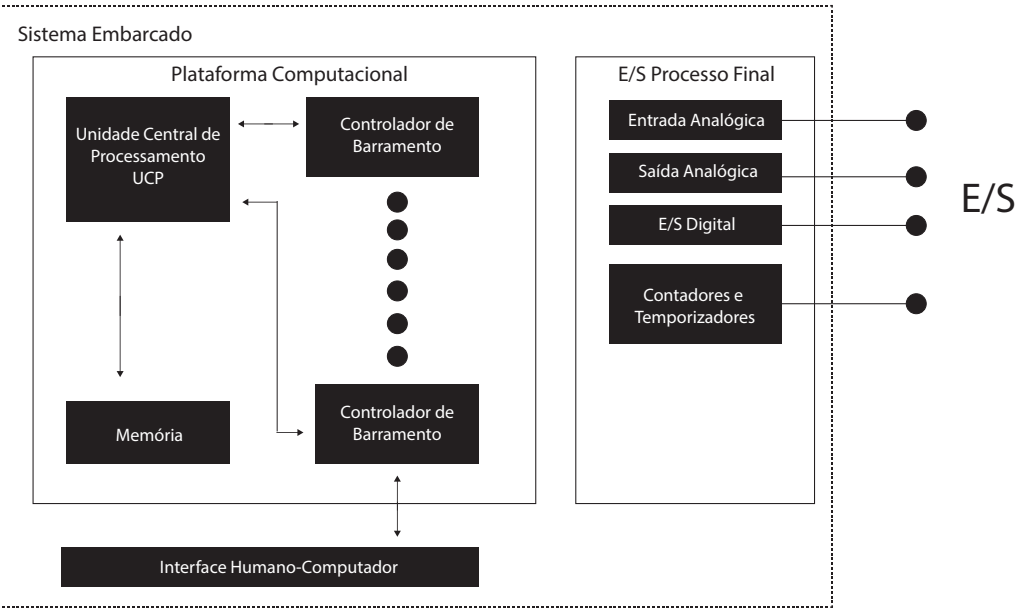

Estes sistemas são compostos de algoritmos de controle que permitem a união de diferentes tecnologias. Sendo assim, plataformas com tais características e com possibilidades de configuração para uso em diferentes contextos são excelentes ferramentas para testes funcionais de produtos que envolvem hardware e software. As plataformas de prototipação rápida, como Arduino, RaspberryPi e BeagleBone são exemplos destes recursos e são amplamente usados atualmente.

Na concepção de Banzi et al. (2005), o Arduíno é uma plataforma de prototipação eletrônica open source baseada em hardware e software flexíveis e fáceis de usar, o que viabiliza a sua utilização na criação de objetos ou ambientes interativos por profissionais de diferentes formações. Projetos no Arduíno incluem um conjunto hardware/software que permite ao projetista simular e se aproximar do comportamento real de um determinado sistema de controle desenvolvido para uma determinada funcionalidade.

Hodges et al. (2012) salientam que o Arduíno tem se tornado o padrão de plataforma aberta para prototipagem física ao permitir a interação com objetos físicos, fazendo-se uso de sensores e atuadores, conectados a
Figura 1. Diagrama de blocos de um Sistema Embarcado. Fonte: Adaptado de Yang (2012). 
microcontroladores. Motivos pelos quais esta plataforma é amplamente aceita na área de computação física (KATO, 2010), pois sua facilidade de aprendizado a torna amplamente utilizada em ensino, em pesquisa e no entretenimento.

A viabilidade de seu uso na fase de projeto está em permitir a definição de características parametrizáveis, e seu propósito geral reside no estabelecimento de ajustes em um dispositivo físico com o envio de sinais de controle para atuadores em reação aos sinais de entrada, provenientes de usuários ou de sensores (WOLF, 2008). Nos processos de fabricação digital, permitem a interação com o ambiente externo e provêm refinamentos em parâmetros do modelo, proporcionado se verificar a viabilidade da aplicação computacional projetada.

\section{METODOLOGIA ADOTADA NO PROCESSO PROJETUAL}

A estruturação deste projeto teve início na disciplina de "Processo de Fabricação Digital como Ferramenta de Projeto”, componente curricular do Programa de Pós-Graduação em Design da UFRGS, por uma equipe constituída por uma Doutoranda em Design - Linha de Pesquisa Projeto de Artefatos, um Mestrando em Design - Linha de Pesquisa em Design Virtual, um aluno especial do Programa de Pós-Graduação em Engenharia Elétrica da UFRGS, e dois professores orientadores, um com Doutorado em Arquitetura e o outro com Doutorado em Engenharia de Produção.

A metodologia adotada para a busca de soluções viáveis e possíveis foi estruturada em quatro fases principais: imersão, ideação, prototipação e validação, sendo estas fases integradas de forma dinâmica, cíclica e contínua, para concepção de soluções possíveis e passíveis de serem realizadas.

A fase de imersão foi direcionada à compreensão e ao entendimento de concepções sobre a experiência e a interação, bem como a importância do contexto de uso para a formação de conceitos. Amparou-se na pesquisa bibliográfica em artigos científicos que fundamentassem as bases teóricas e serviram de alicerce para realização do processo de projeto.

$\mathrm{Na}$ fase de ideação buscou-se adequar o projeto e direcioná-lo, considerando os conhecimentos adquiridos na disciplina de "Processos de Fabricação Digital como Ferramenta de Projeto”. Teve por subsídio as formações de base e o conhecimento dos profissionais envolvidos no projeto para a geração, análise e verificação de alternativas de soluções que proporcionassem uma melhor interação de usuários com a solução proposta e a consequente experiência proveniente desta.

A fase de prototipação constituiu-se da verificação de como componentes digitais poderiam ser integrados e otimizados e da análise dos materiais necessários (microcontroladores, sensores, leds) para que a solução adotada fosse factível. Além disso, o funcionamento destes materiais, para a captura de dados provenientes da interação do usuário, e como os mesmos se apresentavam visualmente aos projetistas, também foi objeto de análise.

$\mathrm{Na}$ fase de validação foram realizados testes de funcionamento dos componentes utilizados e sua precisão, além da reflexão sobre as ideias concebidas e materializadas sobre a solução proposta, a fim de se estabelecer, estruturar e reestruturar as soluções possíveis.

\section{RESULTADOS - REPRESENTAÇÕES DE CONHECIMENTOS}

Representações intermediárias do conhecimento de projetistas provieram da conversa com os materiais por eles manipulados e se formalizaram explicitamente, considerando as etapas anteriormente apresentadas, conforme demonstram as seções subsequentes. 


\section{Imersão - Compreensão do contexto}

Na fase de imersão os projetistas voltam-se ao entendimento do usuário, a fim de compreender suas capacidades e limitações, e do contexto em que a aplicação pode ser utilizada. Foram verificadas formas para explorar interações multimodais, em especial interações táteis de usuários, e na forma de contemplá-las por meio do uso de sensores.

As referências utilizadas para a geração do conceito consideraram a arte de se modelar objetos em barro, o uso de dispositivos que favorecem a entrada de dados multimodais e a similaridade de aplicações computacionais que fazem uso de ambientes tridimensionais e modelagem digital.

Nesta etapa, projetistas buscaram contemplar os preceitos de se projetar para a experiência do usuário, conforme referenciados pela Associação Internacional de Artes Gráficas - AIGA (2010), buscando contemplar uma experiência a um público específico e estabelecer um processo projetual que favoreça a criação de um ambiente que conecte o usuário emocionalmente, agregando valor a sua experiência de uso.

Constitui-se também na análise de ferramentas viáveis de ser aplicadas, considerando o esforço necessário para aprendê-las, o suporte fornecido pelas mesmas à captura de dados da interação e a possibilidade de integração e interoperabilidade entre as ferramentas computacionais a serem utilizadas.

\section{Ideação - Gerando possibilidades}

Tendo em vista que esboços digitais favorecem a criação de modelos e oferecem a possibilidade de se gerar diversas variações do projeto pelo ajuste de parâmetros geométricos, a fase de ideação se voltou para o entendimento relacionado à interação, à experiência de uso da aplicação a ser desenvolvida e à modelagem paramétrica em contextos específicos de uso. Diante disso, a experiência dos projetistas se estabelece a partir da análise de diferentes abordagens, passíveis de serem contempladas, como desenvolvimento de aplicativos para dispositivos móveis, produtos físicos contendo sensores, até o desenvolvimento de software, com diferentes interfaces gráficas, objetivando os conceitos de prototipagem digital.

A partir da verificação, aspectos de convergência e interoperabilidade entre as ferramentas a serem utilizadas (Rhinoceros, Firefly e a plataforma Arduíno) e diante da possibilidade de posterior exportação do código fonte da ferramenta Rhinoceros para uma plataforma para desenvolvimento de software, foram delineados os primeiros esboços de uma solução (vide Figura 2).

Os esboços iniciais, em baixa e média fidelidade, direcionaram-se a ideia de isolar o usuário da complexidade atrelada à modelagem parametrizada e da consequente estruturação de algoritmos pertinentes à prática dos projetistas, tornando as interfaces de usuário (Sistema Computacional Interativo) mais intuitiva e de fácil utilização.

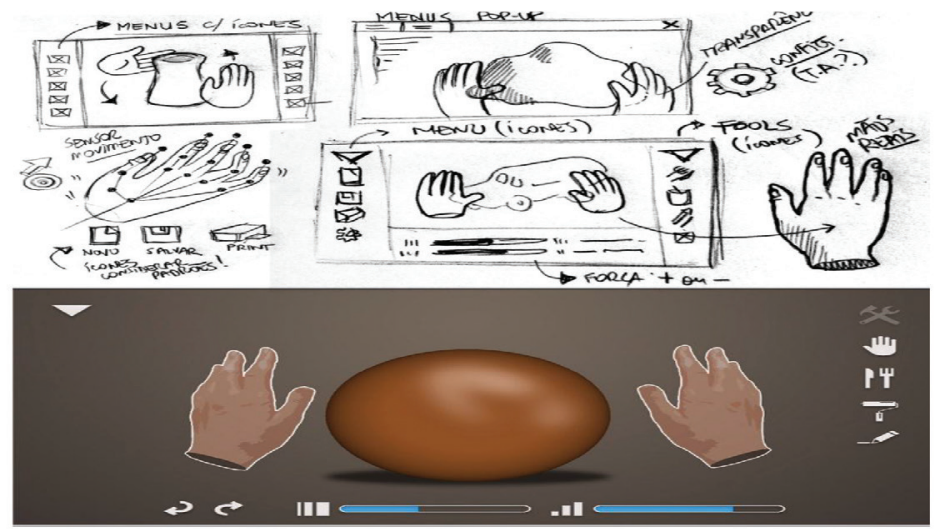

Figura 2. Esboços iniciais para o entendimento e demonstração da solução proposta. Fonte: os autores (2015). 
Diante do exposto, foram estabelecidos alguns requisitos técnicos necessários ao sistema computacional a ser desenvolvido, tais como: capacidade de permitir a interação do usuário de forma tátil, bem como a possibilitar a captura de dados dela provenientes, considerando as oscilações de movimentos táteis do usuário; permitir a realização de ajustes na interface por meio de ícones e menus de forma simples e possibilitando ao mesmo a escolha de objetos a serem modelados; possibilitar a alteração da forma do objeto, a partir dos movimentos do usuário dadas às suas configurações prévias. Esses direcionamentos serviram de base para que a prototipação funcional entre o software e o hardware pudesse ser estabelecida.

\section{Prototipação Funcional - Estruturação de materiais}

A fase de prototipação foi direcionada à preparação do ambiente computacional em termos de hardware e software, a fim de contemplar os requisitos anteriormente definidos. Ferramentas subsidiaram este processo, como a plataforma de prototipagem “Arduíno” (responsável pela captura de dados multimodais), a ferramenta de parametrização geométrica Rhinoceros e os plug-ins Grasshopper e Firefly a ela adicionados para a modelagem geométrica.

A Figura 3 apresenta as interfaces física e digital inicializadas para o monitoramento do hardware e de suas funcionalidades pelos projetistas e permitem a estruturação de relações e correlações de parâmetros que compõem a estrutura algorítmica da aplicação. Com isso, a captura de dados, proveniente do dispositivo físico (3a), torna-se mais intuitiva, tendo em vista que o mapeamento e integração de componentes na ferramenta Rhinoceros se estabelecem de forma similar ao mesmo, facilitando o processo de captura e análise dos dados coletados, bem como a verificação de como eles se apresentam em um determinado momento.

Figura 3. Teste de integração entre dispositivos. Fonte: os autores (2015).

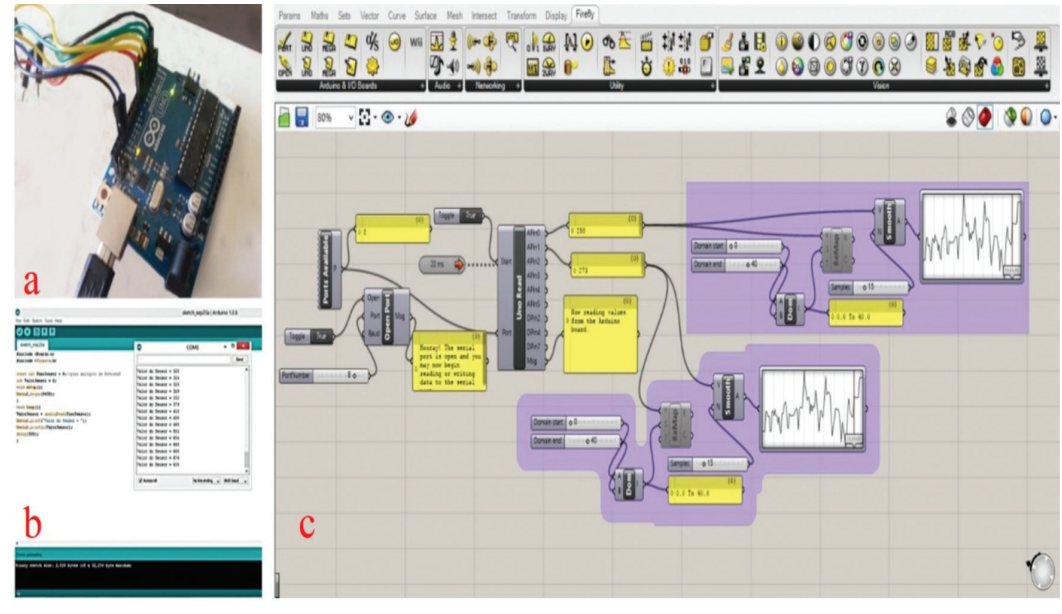

Assim, estas ferramentas subsidiam o processo de fabricação digital e se apresentam como de suma importância para que o produto final (sistema interativo) possa ser desenvolvido, pois o ajuste dos parâmetros, correspondente a coleta de dados, é o que determina a posterior impressão 3D do objeto, modelado pelo usuário.

Para se verificar a correta captura dos dados, componentes adicionais de hardware (leds, sensor ultrassônico e buzzer) foram integrados a um experimento, em decorrência do suporte multimodal que os mesmos oferecem para explorar diferentes sentidos cognitivos dos usuários, conforme demonstra a Figura 4. Os leds retratam a visualização de 
funcionamento da aplicação, o sensor ultrassônico faz referência à distância de um determinado objeto e a sua aproximação, e o buzzer dispara alertas sonoros de acordo com a proximidade de um determinado objeto.

Diante disso, foram realizados testes sobre a aplicação implementada, que agrega em si dispositivos de hardware e software (Figura 4a), considerando a interface de captura de dados da plataforma Arduíno (Figura 3b) e a adequação dos dados coletados com a interface da ferramenta Rhinoceros (Figura 3c) e os respectivos plug-ins (grasshopper e Firefly) a ela integrados.

Conforme demonstra a Figura 4, estes testes foram realizados inicialmente com um objeto estático a fim de se verificar a precisão de captura dos dados pelo sensor de distância, verificando-se o quanto os demais dispositivos (leds e buzzer), acoplados à interface física, estavam sincronizados com esta detecção.
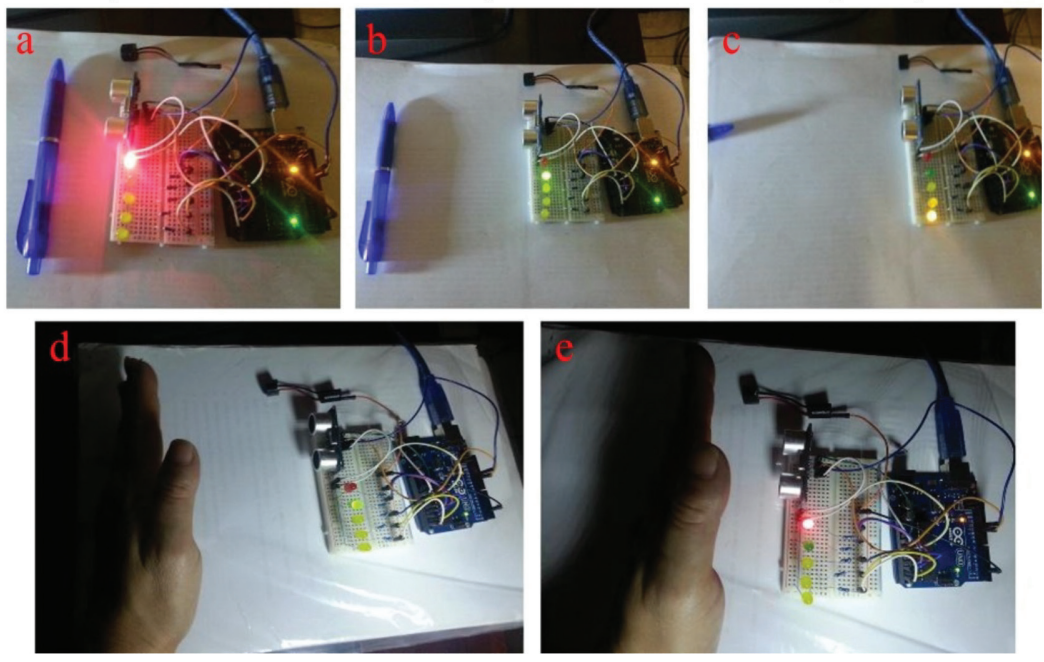

Posteriormente, o experimento foi realizado fazendo-se uso do movimento tátil, visto que a precisão de coleta de dados, a partir do dispositivo físico, pode ser diferenciada pelas oscilações de movimentos. Isso se faz necessário já que os parâmetros obtidos para a modelagem do objeto na interface de usuário (Sistema Interativo) são decorrentes da captura desses dados.

Como pode ser visualizado na Figura 4 (sequência a, b, c, d, e) estes procedimentos remontam a características específicas da experiência de projetistas, tendo em vista que a manipulação destes materiais, bem como a análise do comportamento dos mesmos, permite a estruturação e reestruturação das concepções pré-estabelecidas na fase de ideação e permitem que os mesmos possam refletir sobre sua prática projetual.

Diante disso, soluções distintas são passíveis de ser integradas e aperfeiçoadas, qualificando a experiência do usuário. Retrata também as características de interoperabilidade entre sistemas, referenciadas por Mertins (et al. 2014), tendo em vista a capacidade de comunicação e interação entre as ferramentas selecionadas para o processo projetual, a flexibilidade de manipulação apresentada por elas e o baixo custo associado ao seu uso.

\section{A validação - Melhoria contínua}

A análise de recursos disponíveis e de seu funcionamento, atrelada à convergência e interoperabilidade de materiais, permitiu a apresentação do projeto conceitual de software, bem como a demonstração dos resultados que delinearam o processo projetual, os quais são validados pelos
Figura 4. Testes com materiais para o desenvolvimento do protótipo. Fonte: Elaboração dos autores (2015). 
Figura 5. Protótipo de interface para apresentação do projeto. Fonte: Elaboração dos autores (2015) projetistas a partir da verificação do comportamento do sistema conforme o esperado. Desse modo, a solução proposta se formaliza por meio de um sistema computacional interativo, ou seja, um produto com interface gráfica multimodal para prototipagem digital de objetos, tendo como suporte a modelagem paramétrica, conforme demonstra a Figura 5.
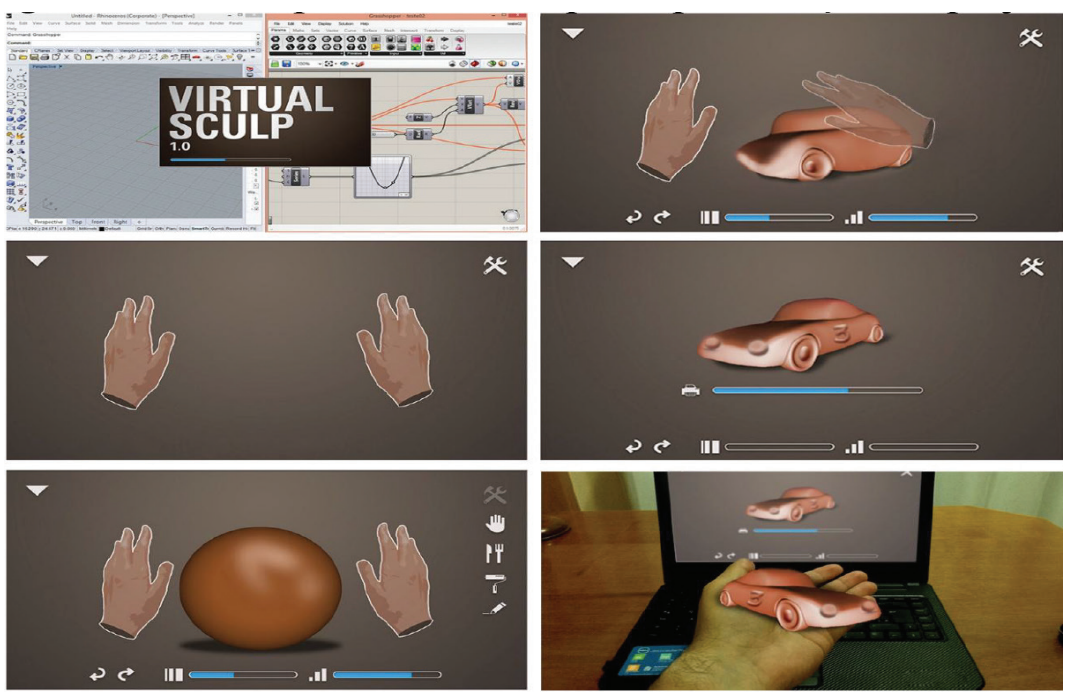

As interações dos usuários são estabelecidas de forma tátil, auxiliadas pela estruturação de componentes que se refletem na interface de usuário, onde a modelagem do objeto, a ser construído pelo usuário, apresenta-se virtualmente amparado por feedbacks que auxiliam o seu uso.

A metáfora utilizada baseou-se no artesanato em argila, com funcionamento semelhante aos softwares de modelagem geométrica utilizados na geração do conceito, porém, de maneira simples, intuitiva e passível de ser manipulada de diferentes formas (interfaces multimodais), o que possibilita a coleta de dados via microcontrolador, amplamente favorecida pela modelagem paramétrica, na qual a manipulação do objeto pelo usuário, de acordo com as configurações por ele definidas, agrega/ subtrai materiais aos objetos por eles manipulados. De posse do objeto delineado e dada a satisfação do usuário , a impressão 3D pode então ser realizada.

\section{CONCLUSÃO}

Os estudos apresentados neste artigo salientam a importância da experiência de projetistas no processo de projeto de soluções computacionais interativas e de como essas experiências se refletem nas interfaces por eles projetadas.

Teve por intuito demonstrar que o resultado do processo projetual não se restringe somente a oferecer ao usuário múltiplas formas de interagir com um determinado objeto, ampliando suas experiências de uso para com a solução proposta. Embora isso, consequentemente, ocorra e seja um dos principais objetivos de se projetar para a experiência, o escopo deste processo perpassa tais pressupostos, tendo em vista que o estudo de ferramentas e a manipulação de materiais, bem como os conhecimentos adquiridos pelos projetistas durante este processo, ampliam suas capacidades técnicocientíficas e visões de mundo, favorecendo o direcionamento de soluções possíveis que se estabelecem a partir da solução proposta.

Assim, o processo projetual delineia-se a partir de uma equipe multidisciplinar com habilidades e, muitas vezes, concepções distintas 
que convergem para a prototipação de soluções possíveis, amparados pela adoção de uma metodologia que delineia o processo de projeto como um todo estruturado. Tarefa que pode se tornar bastante complexa dadas as limitações em termos de conhecimentos/habilidades da equipe e quando se desconsidera a atuação colaborativa e cooperativa a ser mantida pela mesma.

A partir do processo projetual, apresentado neste trabalho, tem-se como resultado uma proposta de solução computacional constituída por um sistema embarcado e validada por testes funcionais que retratam que a modelagem paramétrica favorece o estabelecimento de relações e correlações entre os componentes utilizados na prototipação, e que demonstram que a parametrização auxilia tanto a criação quanto o redesenho de sistemas computacionais interativos, entretanto isto exige uma experiência inicial da equipe, que evolui a cada iteração do protótipo, onde parâmetros são estruturados e reestruturados para compor a solução a ser implementada.

Ressalta-se que os objetivos deste estudo não se voltaram à apresentação de sistema computacional interativo e sua validação por usuários, mas sim demonstrar os resultados provenientes da prática projetual realizada na disciplina "Processos de Fabricação Digital como Ferramenta de Projeto", componente curricular do Programa de Pós-Graduação em Design da Universidade Federal do Rio Grande do Sul - UFRGS. Diante disso, a apresentação de um processo projetual fez-se pertinente para que os aportes teóricos, apresentados neste artigo, fossem identificados de forma mais clara e precisa sobre a prática projetual.

\section{REFERÊNCIAS}

AIGA. American Institute of Graphic Arts. What is experience design? 2001. Disponível em: <http://www.aiga.org/ content.cfm?contentalias=what_is_ed $>$. Acesso em: nov. 2014.AISH, R.; WOODBURY,

R. Multi-level interaction in parametric design. In: Smart Graphics. Springer Berlin Heidelberg, 2005. p.151-162.

BACK, N. et al. Projeto integrado de produtos: planejamento, concepção e modelagem. Barueri: Manole, 2008.

BANZI, M. et al. Site Arduino. 2005 Disponível em: <http://www.arduino.cc $>$. Acesso em: 26 abr. 2014

BENYON, D. Interação humanocomputador. Tradução: Heloisa Coimbra de Souza. 2. ed. São Paulo: Pearson Prentice Hall, 2011.

BROEKMAN, B.; NOTENBOOM, E. Testing embedded software. Pearson Education, 2003

BUCCINI, M. P. R. Introdução ao design experiencial. Recife: Edição do autor, 2008.

ESPINOZA, M.; SCHAEFFER, L. Uso do CAD/ CAE/CAM na Produção de matrizes para processos novos de conformação mecânica. Revista del Instituto de Investigación FIGMMG, v. 7, n. 14, p. 84-91, 2004.

FIALHO, F. A. P. Ciências da cognição Florianópolis: Insular, 2001.
FORLIZZI, J.; DISALVO, C.; HANINGTON, B. On the relationship between emotion, experience and the design of new products. The Design Journal, v. 6, n. 2, p. 29-38, 2003.

FRAGOSO, S. Reflexões sobre a Convergência Midiática. In: Líbero Revista Acadêmica do Programa de PósGraduação da Faculdade Cásper Líbero. São Paulo, ano VIII, n. 15/16, p. 17-21, 2005.

GARRETT, J. J. The elements of user experience: user-centered design for the web and beyond. Pearson Education, 2010.

HALLGRIMSSOM, B. Prototyping and modelmaking for product design. London: Laurence King, 2012.

HASSENZAHL, M. User experience and experience design. In: SOEGAARD, M.; DAM, R. (Eds.). Encyclopedia of humancomputer interaction, 2. ed. Aarhus: The Interaction Design Foundation, 2013.

HAYASHI, E. C. S.; BARANAUSKAS, M. C. C. The affectibility concept in systems for learning contexts. International Journal for e-Learning Security (IJeLS), v. 1, n. 1/2, p. 10-18, 2011.

HENSEL, M.; MENGES, A.;WEINSTOCK. Techniques and Technologies. In: Morphogenetic design, architectural design (AD). Journal of Architectures, 
Michael Editors, Wiley Academy, London, 2008.

HODGES, S.; VILLAR, N.; SCOTT, J.; SCHMIDT, A. A new era for ubicomp development. Pervasive Computing, IEEE, v. 11, n. 1, p. 5-9, 2012.

HOWARD, Z.; MELLES, G. Beyond designing: roles of the designer in complex design projects. In: AUSTRALIAN COMPUTERHUMAN INTERACTION CONFERENCE, 23., 2011, Nova York, NY. Proceedings... Nova York: ACM, 2011. p. 152-155.

JÄÄSKÖ, V.; MATTELMÄKI, T.; YLIRISKU, $S$. The scene of experiences. In: THE GOOD, THE BAD AND THE IRRELEVANT - THE USER AND THE FUTURE OF INFORMATION AND COMMUNICATION TECHNOLOGIES CONFERENCE, 2003, Helsinki, Finland. Proceedings... Helsinki: University of Art and Design Helsinki, 2003. P. 341-345.

KATO, Y. Splish: a visual programming environment for Arduíno to accelerate physical computing experiences. In: INTERNATIONAL CONFERENCE ON CREATING CONNECTING AND COLLABORATING THROUGH COMPUTING, 8., 2010. Proceedings... IEEE Computer Society, 2010. p. 3-10.

KOLAREVIC, B. Architecture in the digital age: design and manufacturing. New York; London: Taylor \& Francis, 2005.

LATOUR, B. A esperança de Pandora: ensaios sobre a realidade dos estudos científicos. In: Filosofia e política. São Paulo: EDUSC, 2001.

LI, S.; XIONG, Z.; LI, T. Distributed cooperative design method and environment for embedded system. In: INTERNATIONAL CONFERENCE ON COMPUTER SUPPORTED COOPERATIVE WORK IN DESIGN, 9., 2005. Proceedings... IEEE, 2005. p. 956-960.

MACHADO, P. D. L. LTS-BT: a tool to generate and select functional test cases for embedded systems. In: ACM SYMPOSIUM ON APPLIED COMPUTING, 2., 2008, Nova York, NY. Proceedings... Nova York: ACM, 2008. p. 1540-1544.

MANZINI, E.; JÉGOU, F. Design degli scenari. In: Design multiverso: appunti di fenomenologia del design, 2004. Milão: Edizioni POLIdesign, p. 180-192, 2004.
MCLELLAN, $H$. Experience design. Cyberpsychology and Behavior, v. 3, n. 1, p. 59-69, 2000

MERTINS, K; BÉNABEN, F; POLER, R.; BOURRIĖRES J-P. Enterprise interoperability vi: interoperability for agility, resilience and plasticity of collaborations. Springer Science \& Business Media, 2014.

ORCIUOLI, A. O impacto das tecnologias de fabricação digital nos processos de design. AU: Arquitetura e Urbanismo, v. 183, 2009. Disponível em: <http://www.revistaau. com.br/arquitetura-urbanismo/183/ imprime141180.asp>. Acesso em: jul. 2013.

PARISI, L. Contagious architecture: computation, aesthetics, and space. Cambridge: MIT Press, 2013.

PEREIRA, A. T. C.; GONÇALVES, B. S. (Org.). Design de hipermídia: processos e conexões. Florianópolis: UFSC/CCE, 2010.

PORTUGAL, C. Design, educação e tecnologia. Rio de Janeiro: Rio Books, 2013.

PREECE, J.; ROGERS, Y.; SHARP, H. Design de interação: além da interação homemcomputador. Tradução: Isabela Gasparini. 3. ed. Porto Alegre: Bookman, 2013.

PRESSMAN, R.; LOWE, D. Engenharia web. Tradução: Daniel Vieira. Rio de Janeiro: LTC, 2009.

SCHEEREN, R.; SPERLING, D. M. Dos diagramas aos parâmetros: transformações no design digital. In: CONGRESSO DA SOCIEDADE IBEROAMERICANA DE GRÁFICA DIGITAL "KNOWLEDGE-BASED DESIGN", 17. Anais... Valparaíso, CL: Universidad Técnica Federico Santa Maria. 2013. p. 262-268

SURI, J. F. The Experience of evolution: developments in design practice. The Design Jounal, v. 6, n. 2, p. 39-48, 2003.

WOODBURY, R. Elements of parametric design. Oxford: Routledge, 2010.

WOLF, M. Computers as components: principles of embedded computing system design. Elsevier, 2012.

YANG, N. Aquisição de dados: I/O para sistemas embarcados. National Instruments, 2012. Disponível em: <http:// www.ni.com/white-paper/7021/en/>. Acesso em: 8 abr. 2014. 